\title{
Pain issues in disorders of consciousness
}

\author{
Camille Chatelle $^{1 *}$, Aurore Thibaut ${ }^{1} *$, John Whyte ${ }^{2}$, Marie Danièle De Val ${ }^{3}$, Steven Laureys ${ }^{1}$, \& Caroline Schnakers ${ }^{1,4}$ \\ ${ }^{1}$ Coma Science Group, Cyclotron Research Centre, University of Liège, Liège, Belgium, ${ }^{2}$ Moss Rehabilitation Research Institute, Elkins Park, PA, USA, \\ ${ }^{3}$ Department of Intensive Care, University Hospital of Charleroi, Charleroi, Belgium, and ${ }^{4}$ Department of Psychology and Department of \\ Neurosurgery, University of California, Los Angeles, CA, USA
}

\begin{abstract}
Background: The assessment of pain and nociception in non-communicative patients with disorders of consciousness (DOC) is a real challenge for clinicians. It is, therefore, important to develop sensitive standardized tools usable at the bedside.

Objectives: This review aims to provide an overview of the current knowledge about pain processing and assessment in patients with DOC.

Methods: A search was performed on PubMed using MeSH terms including vegetative state, unresponsive wakefulness syndrome, minimally conscious state, consciousness disorders, pain, nociception, neuroimaging and pain assessment.

Results: Neuroimaging studies investigating pain processing in patients with DOC and their implication for clinicians are reviewed. Current works on the development of standardized and sensitive tools for assessing nociception are described.

Conclusion: The suggested pain perception capacity highlighted by neuroimaging studies in patients in a MCS and in some patients in a VS/UWS supports the idea that these patients need analgesic treatment and monitoring. The first tool which has been developed to assess nociception and pain in patients with DOC is the NCS. Its revised version represents a rapid standardized and sensitive scale which can be easily implemented in a clinical setting. Complementary pain assessments are also under validation in order to offer more options to clinicians.
\end{abstract}

\section{Keywords}

Minimally conscious state, nociception, pain assessment, unresponsive wakefulness syndrome, vegetative state

\section{History}

Received 2 July 2013

Revised 14 October 2013

Accepted 2 November 2013

Published online 25 July 2014

\section{Introduction}

'Is my relative in pain?' is one of the first questions the clinicians have to face when they are interacting with families of patients with severe brain injury. It is, nevertheless, a difficult question. How to know as, by definition, neither patients in an unresponsive wakefulness syndrome (also known as the vegetative state, VS/UWS [1]), nor patients in a minimally conscious state (MCS [2]) are able to functionally communicate. It is impossible for them to express their feelings or even to use any usual scale (such as the Visual Analogue Scale) to communicate the presence of pain and its subjective intensity [3]. This is, however, one of the most important questions to address, as it has obvious clinical and ethical implications [4]. This review will introduce the neuroimaging findings related to pain perception in patients with disorders of consciousness (DOC) as well as the recent and current investigations performed to develop and validate behavioural protocols (such as the Nociception Coma

\footnotetext{
*Camille Chatelle and Aurore Thibaut contributed equally.
}

Correspondence: Caroline Schnakers, Department of Psychology and Neurosurgery, University of California, Los Angeles, CA 90095-1563. Tel: +1 310-825-8546. E-mail: cschnakers@ucla.edu
Scale-Revised; NCS-R [5]) which will help clinicians in assessing and treating pain in those patients. All the articles mentioned in this review (16 reviews and 13 original articles found on Pubmed) were published between 1991-2013.

\section{Pain processing and disorders of consciousness}

Pain is defined as 'an unpleasant sensory and emotional experience associated with real or potential tissue damage' (p.226) [6], whereas nociception is described as 'an actually or potentially tissue damaging event transduced and encoded by nociceptors' (p.473) [7]. Nociception, hence, refers to the basic processing of a noxious stimulus. It is necessary to pain perception but it will not always lead to a conscious experience [7]. In contrast, pain is a conscious first-person experience, which has to be reported, verbally or nonverbally, to be correctly assessed.

Neuroimaging studies suggest that nociception and pain are mediated by different networks. The stimulation of nociceptors (A- $\delta$ and $\mathrm{C}$ fibres) leads to the transmission of information via the spinothalamic and spinoreticular pathways to the midbrain (i.e. periaqueductal matter) and thalamus. Afterwards, nociceptive information will be transmitted to the cortex. The cortical nociceptive network encompasses the primary (S1) and secondary somatosensory (S2) cortices, as well as the posterior insula (lateral network) 
participating in the sensory-discriminative aspects of pain processing [8-10]. However, the activation of this network is not sufficient to generate the conscious experience of pain which necessitates the cingulate, anterior insula and prefrontal cortices (medial network), considered to be involved in the motivational-affective and cognitive-evaluative aspects of pain processing [11-14]. The involvement of all these areas is what is called the 'pain matrix' [15]. Even though recent studies support the idea that the 'pain matrix' is not only related to pain but is involved in multimodal processing of saliency [16-18], the connectivity within these regions seems to play an important role in conscious perception of pain [19-21].

Using neuroimaging, previous studies aimed to objectify pain perception in patients with DOC. Laureys et al. [22] investigated central processing of noxious stimuli by using $\mathrm{H}_{2} \mathrm{O}$ positron emission tomography (PET) imaging in postcomatose patients. An increase of metabolism was observed in the midbrain, contralateral thalamus and $\mathrm{S} 1$ in response to an electrical stimulation applied to the median nerve of the wrist in a group of 15 patients in a VS/UWS. The activated S1 was functionally disconnected from S2, bilateral posterior parietal, premotor, polysensory superior temporal and prefrontal cortices as compared to 15 healthy controls. The severely impaired functional connectivity in cortico-cortical pathways suggests that, in patients in a VS/UWS, the activation of the primary cortex seems to be isolated from higher-order associative cortices, reducing the probability that painful stimuli are experienced in an integrated and conscious manner. As regards patients in a MCS, Boly et al. [23] reported brain activation similar to controls in response to noxious stimuli encompassing not only the midbrain, thalamus and S1, but also S2, insular, posterior parietal and posterior part of the anterior cingulate cortex posterior part of the anterior cingulate cortex (ACC) [23] (Figure 1). The activation of these areas (and, particularly, ACC and insula) suggests that patients in a MCS may perceive the unpleasant aspect of painful stimuli $[14,24]$. Moreover, intact connectivity between primary and associative cortices has also been observed in these patients, suggesting the existence of an integrated and distributed neural processing which makes plausible the existence of conscious pain perception in this population.

It is important to stress that, despite these neuroimaging studies, it is still unclear whether all patients in a VS/UWS are unable to feel pain. Kassubek et al. [25] performed a $\mathrm{H}_{2} \mathrm{O}$ PET scan when administering an electrical noxious stimulus in seven post-anoxic patients in a VS/UWS. A group analysis revealed a pain-induced activation in $\mathrm{S} 1$ but also in $\mathrm{S} 2$ and in the cingulate cortex which is involved in the affective pain network. More recently, de Tommaso et al. [26] used nociceptive-specific laser evoked potentials known to be related to generators, such as the ACC, and have reported a response (with a longer latency) in three patients in a VS/UWS. Furthermore, two other studies reported an activation of the affective pain network (i.e. ACC and insula) in $30 \%$ of patients in a VS/UWS in response to noxious stimulation as well as pain cries [27, 28] (Figure 1). The last study, nevertheless, also showed, in parallel to previous findings, that the connectivity within the whole pain network was significantly decreased as compared to patients in a MCS [29]. Even though this suggests an altered perception in patients in a VS/UWS, the activation of the affective pain network might denote the presence of residual pain perception

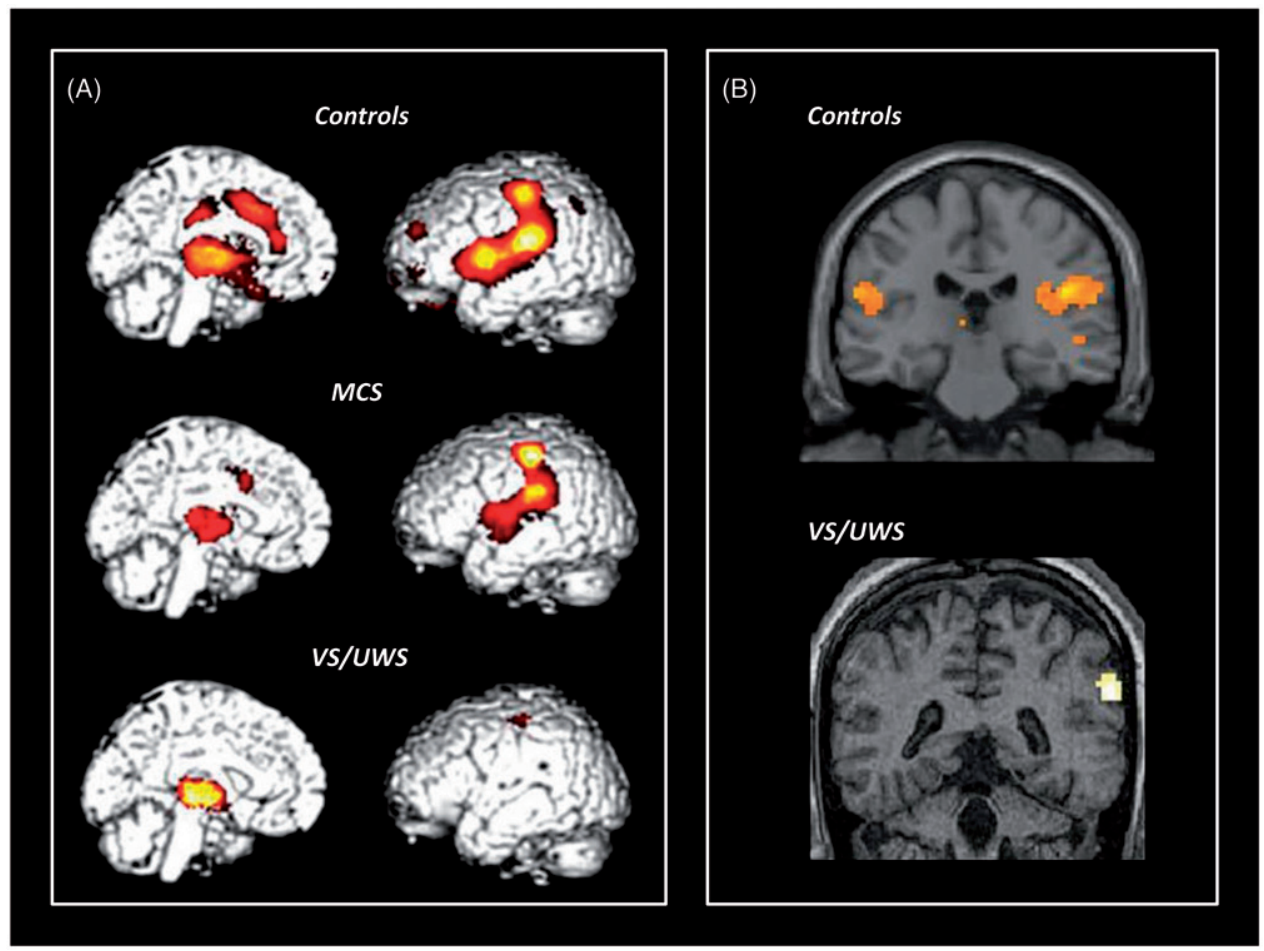

Figure 1. Cerebral activation to noxious stimulation in patients in a VS/UWS and in a MCS as compared to healthy controls. Panel A illustrates, in yellow/red, brain regions activated during noxious stimulation in healthy controls, in patients in a minimally conscious state (MCS) and in a vegetative state/unresponsive wakefulness syndrome (VS/UWS) (adapted from [37]). Panel B illustrates the unusual activation of secondary somatosensory area in response to noxious stimulation in one patient diagnosed as being in a VS/UWS (adapted from [27]). 
in some of those patients. As a minority of patients behaviourally diagnosed as VS/UWS have previously shown brain activation in response to active cognitive tasks [30-32], it is also plausible to assume that a percentage of patients who do not show behavioural signs of consciousness may be able to perceive external stimuli, such as pain. This underlines the importance of considering the potential experience of pain in all patients with DOC and to develop tools to appropriately assess and treat pain in those patients.

\section{Pain management in disorders of consciousness}

Pain management in patients with DOC remains challenging, the assessment being limited by the absence of communication. Several scales have been developed and validated to detect pain in non-communicative patients, such as newborns [33] or patients with dementia [34]. However, until recently, no scale has been developed to assess pain in patients with DOC. Patients in a VS/UWS can demonstrate responses, such as grimaces, cries, tachycardia or tachypnea, which may follow pathological activation of sub-cortical pathways and may be unrelated to painful stimuli [35]. It is, therefore, necessary to develop sensitive standardized tools to accurately assess responses related to nociception and pain in this population.

\section{The nociception coma scale}

Recently, a new scale has been developed to assess nociception and pain in patients with DOC, the NCS [36]. The term 'nociception' was chosen for two reasons. First, the NCS aimed to assess both patients in a VS/UWS and in a MCS and is, therefore, assessing responses underlying both low-level brain processing related to nociception (as observed in a majority of patients in a VS/UWS) and high-level brain processing related to pain (as observed in patients in a MCS). Second, as pain is a subjective experience, it is difficult to use this term when no self-report is available.

The first version of the NCS [36] was based on pre-existing pain scales developed for non-communicative patients with advanced dementia [34] and newborns [33]. It consisted of four sub-scales assessing motor, verbal and visual responses to noxious stimuli as well as facial expression. Its total score ranged from 0-12. Initially, breathing responses were also assessed but later discarded due to the difficulty to reliably assess breathing patterns in patients not benefitting from respiratory monitoring devices (which is often the case in a sub-acute setting) [37]. Previous studies have also shown that physiological parameters seem insufficiently sensitive for pain assessment [38], as it was found to be unrelated to selfreports $[39,40]$ and to fluctuate similarly in response to either non-noxious or noxious stimulation [41]. Stress, medication, medical complications but also brain lesions affecting autonomic functions may also influence these parameters and bias the assessment [42, 43].

The NCS has been validated in patients from intensive care, neurology/neurosurgery units, rehabilitation centres and nursing homes. In a first study including 48 patients (28 VS/UWS and $20 \mathrm{MCS}$; age range $=20-82$ years; 17 of traumatic aetiology), behavioural responses to a noxious stimulus (i.e. nail bed pressure) were scored by two raters using the NCS and four other scales previously validated for non-communicative patients (i.e. the Neonatal Infant Pain Scale [44], the Faces, Legs, Activity, Cry, Consolability pain assessment tool [45], the Pain Assessment In Advanced Dementia Scale [46] and the Checklist of Non-verbal Pain Indicators [47]). Results demonstrated good inter-rater reliability and good concurrent validity for the NCS total scores and sub-scores. Furthermore, as compared to the other pain scales, the NCS showed a broader score range and a better sensitivity to clinical diagnosis, patients in a VS/UWS showing lower scores than those in a MCS, which suggests that the NCS is particularly relevant for the evaluation of pain in patients with DOC. Although those results support the good psychometric properties of the scale, they do not ensure that the observed behavioural changes were actually specific to the noxious stimulus administered as they may also occur spontaneously or in response to non-noxious stimuli.

A second study including 64 patients (27 VS/UWS and 37 MCS; age range $=20-82$ years; 22 of traumatic aetiology) was, therefore, performed in order to compare NCS scores observed at rest, in response to a non-noxious stimulus (i.e. tap on the shoulders) and in response to a noxious stimulus (i.e. nail bed pressure) [5]. Results showed that NCS total scores as well as motor, verbal and facial sub-scores were significantly higher in response to a noxious stimulus than at rest or in response to a non-noxious stimulus, reflecting the good sensitivity of the scale. However, no difference could be observed between noxious and nonnoxious conditions for the visual sub-scores, suggesting that this sub-scale was not specific to nociception. The authors, therefore, decided to propose a new version excluding the visual sub-scale, the Nociception Coma Scale-Revised (NCS-R) (see Table I) [5]. Based on this version, a cut-off score of 4 has been defined as a potential clinical threshold for detecting pain in patients with DOC (see the sub-section below). Indeed, this cut-off score seems to be the best one for differentiating noxious from non-noxious stimuli, with a sensitivity of $73 \%$, a specificity of $97 \%$ and an accuracy of $85 \%$.

Finally, a recent neuroimaging study has investigated whether the NCS-R was related to brain areas involved in the so-called pain matrix [48]. Using a 18-Fluoro-deoxyglucose PET scan, a significant correlation was found between NCS-R total scores and brain metabolism in the ACC. Those results

Table I. The nociception coma scale-revised.

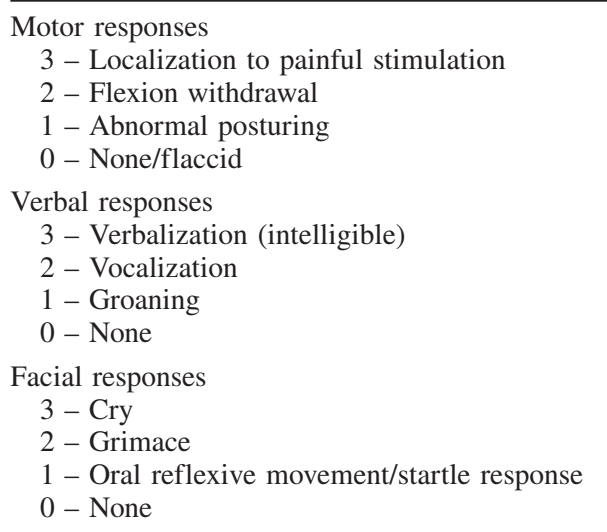


suggest that the NCS-R is at least partially related to cortical pain processing and, hence, may constitute an appropriate behavioural tool to assess, monitor and treat nociception and pain in non-communicative patients with DOC. Further studies should, nevertheless, investigate whether the scale is related to the connectivity of the ACC with the rest of the pain matrix, as it has been shown that cortico-cortical connectivity is related to consciousness and, hence, to conscious pain perception (see above 'Pain processing and disorders of consciousness').

\section{How to use it}

The administration of the NCS-R should be done in all patients who are in a VS/UWS or in a MCS, especially those who present a documented potential pain (e.g. polytraumatic injuries, decubitus ulcers, severe spasticity or arthralgia; see clinical manual in supplementary material). It is also crucial to obtain any information about pre-existing pain (e.g. osteoarthritis, rheumatoid arthritis or gout) from the family or the legal surrogate before starting using the scale. Behavioural signs of pain at rest (e.g. grimaces or cries) can be taken into consideration. However, those responses could be unrelated to pain (e.g. pathological activation of sub-cortical areas leading to constant but not appropriate cries [49]) and has, therefore, to be replicated in a pain-related condition (i.e. mobilization/palpation).

The NCS-R should be scored at rest in order to observe the spontaneous responses presented by the patient (assessed with eyes opened) during at least 1 minute before starting potentially painful care and/or stimulating a potentially painful area. Behavioural responses observed during the care and/or stimulation are then scored with the NCS-R. The highest score obtained for each sub-scale is summed to obtain the total score. In case of a documented cause of potential pain, the NCS-R should be administered before and after treatment. It is essential to assess simultaneously the patient's level of consciousness in order to avoid over-medication. Indeed, it is likely that the administration of narcotic or other sedating analgesics will decrease the presence of pain behaviours, but also the presence of signs of consciousness, as those medications may have an impact on alertness and vigilance in patients who already show deficits in those domains. A balance has, therefore, to be found between under-treatment and over-treatment in revising pain treatment regularly. Considerations should be given to non-sedating medications or to medications with reversible effects (e.g. opiate reversal with naltrexone) whenever there is question of medication effects vs. ongoing deterioration of neurological status.

In this context, the NCS-R may constitute a helpful instrument for monitoring pain behaviour on a daily basis. In the absence of documented conditions likely to produce pain, a sudden increase of the NCS-R total score during the hospitalization independent from an improvement in the level of consciousness will alert the clinician of the potential presence of pain. Additional investigations may then be performed to identify its origin/localization (e.g. by using mobilization/palpation). Finally, even though additional investigations are needed to make the NCS-R clinically valid, a score equal or superior to 4 observed during care could be used as an indicator of potential pain to start the administration of analgesics.

\section{Further validation}

Previous investigations aimed to develop and validate the first scale assessing nociception and pain in patients with DOC. Now that the psychometric qualities of this tool (such as its validity and its sensitivity) have been tested using experimental noxious stimuli, a clinical validation of the scale is needed. A collaboration between the University Hospital of Charleroi and the Cyclotron Research Centre in Liège, Belgium aiming to confirm the interest of the NCS-R in cases of clinical pain has just been completed. Patients with documented potential pain (e.g. fracture, decubitus ulcers or spasticity) have received an analgesic treatment tailored to the type of pain presented and have been re-evaluated within 24 hours. Both assessments have been performed during nursing care in order to compare similar conditions. The Glasgow Coma Scale (GCS) has also been administered before and after treatment in order to observe fluctuations in consciousness. The GCS has been chosen as this scale was already implemented in the units where the study was conducted. If the NCS-R is a sensitive clinical tool, its total score should decrease after the administration of the treatment. Such a study should also allow one to test if the cut-off score of 4 is really related to the presence of pain. If this is the case, a score equal or superior to 4 should be found in patients with documented potential pain. Finally, if the treatment is efficient, it should decrease pain while maintaining the patient's level of consciousness. In that context, a decrease on the NCS-R total score should not be accompanied by a significant decrease of the GCS total score and, hence, of the consciousness level. As the type of pain and the exact treatment administered are documented, preliminary guidelines could be discussed with regards to the type of treatment to implement in order to avoid over- or under-treatment of pain in patients with DOC. Therefore, besides the aim to demonstrate the clinical value of the NCS-R, this study could lead to the development of clinical guidelines which currently do not exist and which should address, in the future, important issues such as specific analgesic treatments depending on the type of pain (e.g. baclofen for spasticity), potential nonpharmacological treatments and prevention of pain.

\section{Other approaches under development for assessment of nociception}

Clinicians caring for patients with DOC have varied reasons for being concerned about pain. Concern for the patient's suffering and, hence, about the subjective experience of pain, is an ethical responsibility of clinicians and can influence treatment decisions [4]. As mentioned previously, current studies aim to investigate whether those patients are capable of this subjective suffering [22, 23, 27]. However, clinicians should also be concerned about nociception, regardless of the patient's subjective experience, since detection of nociceptive stimuli may signal medical problems that require attention, such as undiagnosed fractures or kidney stones. Given the well-documented prevalence of diagnostic error about the 
state of consciousness and given the fact that few patients have been studied in great details [30,32, 50], most clinicians should not assume the absence of subjective suffering [51]. When the level of pain awareness is in doubt, the prevention of pain should always prevail. Moreover, clinicians caring for such patients soon after injury have the greatest reason to be concerned about detecting medical complications, but are the least confident of the patient's state of consciousness on a given day because of the ongoing evolution. Thus, particularly in the relatively early period after injury, presence of nociceptive stimuli is of greater concern than the question of subjective pain. As a corollary, a method of assessing the presence of nociceptive stimuli through behavioural and/or physiologic responses for use in this context should not hinge on the patient's state of consciousness.

The human response to nociceptive stimuli is complex, including autonomic responses and reflexive and voluntary motor responses [21]. Although patients in the VS/UWS do not demonstrate the voluntary aspects of this repertoire (e.g. grasping the arm of an examiner), they generally still display a wide range of responses to nociception. Such patients often have autonomic responses to skin lesions, urinary retention or range of motion exercises for example, sometimes even in exaggerated form, as part of the Paroxysmal Sympathetic Hyperactivity [52]. They may also display facial grimacing, moaning, tearing, increases in tonic posturing and thrashing limb movements.

Currently, the NCS-R is the only measure of nociception and pain developed specifically for individuals with DOC and which has any validation data available. However, this tool may have limitations for use in the context discussed above. One is that the highest number of points that can be scored depends on the patient's state of consciousness, as well as the presence or severity of nociceptive stimulation. Another is that it has a relatively small point range, particularly if one discounts the items requiring consciousness. Thus, it may be useful to develop a measurement tool with a wider range of items to maximize sensitivity to the intensity of nociception. More widespread clinical validation will be needed for any similar scale, which is the subject of an ongoing research project discussed below.

Many of the observational tools existing for other populations, in whom assessment of pain and nociception is difficult, also include content that would vary in its presence by level of consciousness (e.g. vocal complaints, localization to pain, cries). In addition, some focus on deviations from wellestablished patterns of behaviour, which makes them hare to use in an acute setting or by caregivers not familiar with the patient [36]. There is also reason to be concerned about transferring observational pain scales from other populations to patients with DOC due to brain injury because of the prevalence of agitated behaviour in patients beginning to regain consciousness. Agitation may also involve facial grimacing, moaning and limb movements for example [53]. While it is believed that the presence of nociceptive stimuli can prompt outbursts of agitation, it is also believed that agitation can appear as a primary syndrome related to brain injury, in the absence of noxious stimulation. This latter assumption, however, is difficult to prove, since one can never be completely certain about the absence of nociception.
In preparation for a research project on validating an observational measure of nociception, the Moss Rehabilitation Research Institute in Elkins Park, PA, conducted informal interviews with six nurses experienced in caring for patients with DOC in an acute inpatient rehabilitation setting and three family members, two accompanying patients to the acute rehabilitation hospital and one caring for a family member at home. There was consensus among the nurses that a structured rating tool for use in DOC related to brain injury would be useful. They reported that uncertainty about the presence and source of nociceptive stimuli in these patients is a frequent concern and that, when physicians order 'as needed' analgesics, they have very little to guide their administration, particularly when the patient is new to them. As expected, they experienced the greatest uncertainty in patients who displayed frequent agitation. Family members, too, reported worrying about whether their loved one was in pain and not being sure about the significance of various responses observed. Caregivers and nurses alike reported that they felt increasingly confident over time in judging behavioural indicators of nociception, much as a parent of a newborn learns to recognize certain characteristic cries. But also, like new parents, some of these behaviours remain ambiguous and some that are used as indicators may not be valid.

The clinical dilemma faced by nurses and family members is whether certain physiologic (e.g. rise in heart rate or blood pressure, dilation of pupils) or behavioural indicators (e.g. facial grimaces, moaning, restless movement) signify the presence of some underlying condition producing nociception, when that stimulus may be unknown. In contrast, the validation of the NCS-R to date has focused on the detection of behavioural responses to experimental stimuli known to be noxious in nature [5]. Thus, other authors sought to develop a clinical measure of the presence of nociceptive conditions and to attempt to validate it for clinical use.

This ongoing study, a collaboration between investigators at Moss Rehabilitation Research Institute in the US and Glostrup Hospital in Denmark, aims to develop and validate a unidimensional quantitative observational scale of the severity of nociceptive conditions for use in patients with brain injury who are unable to communicate about pain due to DOC. In the first step of this research, they have assembled a broad set of behavioural and physiologic indicators, like those mentioned above, from review of other similar scales and the prior experience of the investigators. They are in the process of collecting observational ratings from a large number of patients at both sites. Although conditions, such as hypertension and tachycardia due to dehydration, may confound the use of these physiologic indicators in the scale, the investigators have chosen to collect data on them for item response theory (IRT) analysis and to discard them based on those results if needed. IRT is a method that identifies rating items that are all measures of an underlying dimension (in this case nociception) and locates them on that dimension (in this case, reflecting intensity of nociception where, hypothetically, a frown might reflect a lower intensity than a scream). Participants are rated on multiple occasions in various environmental contexts (e.g. at rest, during 
Physical Therapy) and on different doses of analgesia. Known conditions likely to produce nociception, such as fractures and recent surgeries, are coded for all patients, as well as the presence of agitation.

Data will first be analysed with IRT methods to develop a unidimensional scale of the intensity of nociception and to understand the relationship between that construct and the agitation measure. Patient scores on the revised nociception instrument will be validated against other clinical measures. Specifically, the hypotheses are that scores on the refined measure will be related to the presence or absence of known conditions likely to produce nociception, will vary with analgesic dose and will be higher during gross movement activities than at rest after repositioning.

\section{Conclusion}

In acute as in chronic stages, several conditions, such as polytraumatic injuries, decubitus ulcers, severe spasticity or arthralgia, are likely to induce pain, especially during care and mobilization [54]. The suggested pain perception capacity highlighted by neuroimaging studies in patients in a MCS and in some patients in a VS/UWS supports the idea that these patients need analgesic treatment and monitoring. The first tool, which has been developed to assess nociception and pain in patients with DOC, is the NCS. Its revised version, the NCS-R, represents a rapid, standardized and sensitive scale, which can be easily implemented in acute and sub-acute units. Complementary pain assessments are also currently under validation in order to offer more options to clinicians. Even though it is crucial to have tools for detecting and treating acute pain, another challenge in the future will be to develop or adapt existing scales to detect chronic pain (i.e. pain lasting longer than 3-6 months) in patients with DOC. Indeed, it has been shown that the prevalence of chronic pain is high (i) after a traumatic brain injury (as $50 \%$ of patients with mild-to-moderate injury report recurrent headaches), (ii) after a non-traumatic brain injury (as thalamic lesion may lead to chronic pain) and (iii) in presence of recurrent bedsores, severe spasticity or uncomfortable deformities [55]. Chronic pain has also been related to motivational-affective and cognitive-evaluative aspects of pain processing and to suffering. Suffering involves a complex cognitive-affective phenomenon and implies not only a negative emotional response to pain experience, but also the ability to remember that particular experience or set of experiences. As some patients diagnosed as being in a MCS or in a VS/UWS have previously shown complex remnant cognitive functioning (e.g. attention or working memory) [30-32], studies have to be performed in order to better characterize the cognitive pattern existing in this population and, therefore, the potential suffering experienced by these patients. Therefore, additional investigations will have to be performed in order to develop a complete battery of valid and sensitive measures for clinicians to efficiently detect nociception and pain (both acute and chronic) in patients with severe brain injury. Such tools will certainly allow clinicians to prevent and treat pain in this challenging population, but they will also lead to develop guidelines that are currently inexistent and, hence, crucially needed.

\section{Declaration of interest}

The authors report no conflicts of interest. This study was supported by the National Funds for Scientific Research (FNRS), Action de Recherche Concertée, James S. McDonnell Foundation, Mind Science Foundation, University of Liège and the National Institute on Disability and Rehabilitation Research (Grant \# H133A070040).

\section{References}

1. Laureys S, Celesia GG, Cohadon F, Lavrijsen J, Leon-Carrion J, Sannita WG, Sazbon L, Schmutzhard E, von Wild KR, Zeman A, et al. Unresponsive wakefulness syndrome: A new name for the vegetative state or apallic syndrome. BMC Medicine 2010;8:68.

2. Giacino J, Ashwal S, Childs N, Cranford R, Jennett B, Katz D, Kelly J, Rosenberg J, Whyte J, Zafonte R. The minimally conscious state: Definition and diagnostic criteria. Neurology 2002;58: 349-353.

3. Huskisson EC. Measurement of pain. Journal of Rheumatology 1982;9:768-769.

4. Demertzi A, Racine E, Bruno M-A, Ledoux D, Gosseries O, Vanhaudenhuyse A, Thonnard M, Soddu A, Moonen G, Laureys S. Pain perception in disorders of consciousness: Neuroscience, clinical care, and ethics in dialogue. Neuroethics 2013;6:37-50.

5. Chatelle C, Majerus S, Whyte J, Laureys S, Schnakers C. A sensitive scale to assess nociceptive pain in patients with disorders of consciousness. Journal of Neurology, Neurosurgery \& Psychiatry 2012;83:1233-1237.

6. Task Force on Taxonomy of the International Association for the Study of Pain. Classification of chronic pain: Descriptions of chronic pain syndromes and definitions of pain terms. Seattle, WA: IASP Press 1994.

7. Loeser JD, Treede RD. The Kyoto protocol of IASP Basic Pain Terminology. Pain 2008;137:473-477.

8. Ploner M, Gross J, Timmermann L, Schnitzler A. Cortical representation of first and second pain sensation in humans. Proceedings of the National Academy of Science USA 2002;99: 12444-12448.

9. Lockwood PL, Iannetti GD, Haggard P. Transcranial magnetic stimulation over human secondary somatosensory cortex disrupts perception of pain intensity. Cortex 2012;49:2201-2209.

10. Ploner M, Schmitz F, Freund HJ, Schnitzler A. Parallel activation of primary and secondary somatosensory cortices in human pain processing. Journal of Neurophysiology 1999;81:3100-3104.

11. Sikes RW, Vogt BA. Nociceptive neurons in area 24 of rabbit cingulate cortex. Journal of Neurophysiology 1992;68:1720-1732.

12. Peyron R, Garcia-Larrea L, Gregoire MC, Convers P, Richard A, Lavenne F, Barral FG, Mauguiere F, Michel D, Laurent B. Parietal and cingulate processes in central pain. A combined positron emission tomography (PET) and functional magnetic resonance imaging (fMRI) study of an unusual case. Pain 2000;84:77-87.

13. Vogt BA. Pain and emotion interactions in subregions of the cingulate gyrus. Nature Reviews Neuroscience 2005;6:533-544.

14. Shackman AJ, Salomons TV, Slagter HA, Fox AS, Winter JJ, Davidson RJ. The integration of negative affect, pain and cognitive control in the cingulate cortex. Nature Reviews Neuroscience 2011; 12:154-167.

15. Ingvar M. Pain and functional imaging. Philosophical Transactions of the Royal Society of London. Series B, Biological sciences 1999; 354:1347-1358

16. Mouraux A, Diukova A, Lee MC, Wise RG, Iannetti GD. A multisensory investigation of the functional significance of the “pain matrix". Neuroimage 2011;54:2237-2249.

17. Moulton EA, Pendse G, Becerra LR, Borsook D. BOLD responses in somatosensory cortices better reflect heat sensation than pain. Journal of Neuroscience 2012;32:6024-6031.

18. Ronga I, Valentini E, Mouraux A, Iannetti GD. Novelty is not enough: Laser-evoked potentials are determined by stimulus 
saliency, not absolute novelty. Journal of Neurophysiology 2013; 109:692-701.

19. Massimini M, Boly M, Casali A, Rosanova M, Tononi G. A perturbational approach for evaluating the brain's capacity for consciousness. Progress in Brain Research 2009;177:201-214.

20. Owen AM, Schiff ND, Laureys S. A new era of coma and consciousness science. Progress in Brain Research 2009;177: 399-411.

21. Schnakers C, Chatelle C, Demertzi A, Majerus S, Laureys S. What about pain in disorders of consciousness? AAPS Journal 2012;14: 437-444.

22. Laureys S, Faymonville M, Peigneux P, Damas P, Lambermont B, Del Fiore G, Degueldre C, Aerts J, Luxen A, Franck G. Cortical processing of noxious somatosensory stimuli in the persistent vegetative state. Neuroimage 2002;17:732-741.

23. Boly M, Faymonville ME, Schnakers C, Peigneux P, Lambermont B, Phillips C, Lancellotti P, Luxen A, Lamy M, Moonen G, et al. Perception of pain in the minimally conscious state with PET activation: An observational study. The Lancet Neurology 2008;7: 1013-1020.

24. Bingel U, Quante M, Knab R, Bromm B, Weiller C, Buchel C. Subcortical structures involved in pain processing: Evidence from single-trial fMRI. Pain 2002;99:313-321.

25. Kassubek J, Juengling FD, Els T, Spreer J, Herpers M, Krause T, Moser E, Lucking $\mathrm{CH}$. Activation of a residual cortical network during painful stimulation in long-term postanoxic vegetative state: A 15O-H2O PET study. Journal of Neurological Sciences 2003; 212:85-91.

26. de Tommaso M, Navarro J, Ricci K, Lorenzo M, Lanzillotti C, Colonna F, Resta M, Lancioni G, Livrea P. Pain in prolonged disorders of consciousness: Laser evoked potentials findings in patients with vegetative and minimally conscious states. Brain Injury 2013;27:962-972.

27. Markl A, Yu T, Vogel D, Muller F, Kotchoubey B, Lang S. Brain processing of pain in patients with unresponsive wakefulness syndrome. Brain and Behavior 2013;3:95-103.

28. Yu T, Lang S, Vogel D, Markl A, Muller F, Kotchoubey B. Patients with unresponsive wakefulness syndrome respond to the pain cries of other people. Neurology 2013;80:345-352.

29. Kotchoubey B, Merz S, Lang S, Markl A, Muller F, Yu T, Schwarzbauer C. Global functional connectivity reveals highly significant differences between the vegetative and the minimally conscious state. Journal of Neurology 2013;260:975-983.

30. Monti MM, Vanhaudenhuyse A, Coleman MR, Boly M, Pickard JD, Tshibanda L, Owen AM, Laureys S. Willful modulation of brain activity in disorders of consciousness. New England Journal of Medicine 2010;362:579-589.

31. Schnakers C, Perrin F, Schabus M, Hustinx R, Majerus S, Moonen G, Boly M, Vanhaudenhuyse A, Bruno MA, Laureys S. Detecting consciousness in a total locked-in syndrome: An active event-related paradigm. Neurocase 2009;15:271-277.

32. Cruse D, Chennu S, Chatelle $\mathrm{C}$, Bekinschtein T, Fernández-Espejo D, Junqué C, Pickard J, Laureys S, Owen A. Bedside detection of awareness in the vegetative state. The Lancet 2011;378:2088-2094.

33. American Geriatrics Society. The management of persistent pain in older persons. Clinical Practice Guideline 2002;50:S205-S224.

34. Hummel P, van Dijk M. Pain assessment: Current status and challenges. Seminars in Fetal \& Neonatal Medicine 2006;11: 237-245.

35. The Multi-Society Task Force on PVS. Medical aspects of the persistent vegetative state (2). The Multi-Society Task Force on PVS. New England Journal of Medicine 1994;330:1572-1579.

36. Schnakers C, Chatelle C, Vanhaudenhuyse A, Majerus S, Ledoux D, Boly M, Bruno MA, Boveroux P, Demertzi A, Moonen G, et al. The Nociception Coma Scale: A new tool to assess nociception in disorders of consciousness. Pain 2010;148:215-219.

37. Schnakers C, Chatelle C, Majerus S, Gosseries O, De Val M, Laureys S. Assessment and detection of pain in noncommunicative severely brain-injured patients. Expert Review of Neurotherapeutics 2010;10:1725-1731.

38. Herr K, Coyne PJ, McCaffery M, Manworren R, Merkel S. Pain assessment in the patient unable to self-report: Position statement with clinical practice recommendations. Pain Management Nursing 2011;12:230-250.

39. Gelinas C, Arbour C. Behavioral and physiologic indicators during a nociceptive procedure in conscious and unconscious mechanically ventilated adults: Similar or different? Journal of Critical Care 2009;24:628 e7-17.

40. Gelinas C, Johnston C. Pain assessment in the critically ill ventilated adult: Validation of the Critical-Care Pain Observation Tool and physiologic indicators. Clinical Journal of Pain 2007;23: 497-505.

41. Young J, Siffleet J, Nikoletti S, Shaw T. Use of a Behavioural Pain Scale to assess pain in ventilated, unconscious and/or sedated patients. Intensive \& Critical Care Nursing 2006;22:32-39.

42. Buttner W, Finke W. Analysis of behavioural and physiological parameters for the assessment of postoperative analgesic demand in newborns, infants and young children: A comprehensive report on seven consecutive studies. Paediatric Anaesthesia 2000;10: 303-318.

43. Herr KA, Garand L. Assessment and measurement of pain in older adults. Clinics in Geriatric Medicine 2001;17:457-478.

44. Lawrence J, Alcock, D, McGrath P, Kay J, MacMurray SB, Dulberg C. The development of a tool to assess neonatal pain. Neonatal Network 1993;12:59-66.

45. Merkel SI, Shayevitz JR, Voepel-Lewis T, Malviya S. The FLACC: A behavorial scale for scoring postoperative pain in young children. Pediatric Nursing 1997;23:293-297.

46. Warden V, Hurley AC, Volicer L. Development and psychometric evaluation of the Pain Assessment in Advanced Dementia (PAINAD) Scale. Journal of the American Medical Directors Association 2003:4:9-15.

47. Feldt KS. The checklist of nonverbal pain indicators. Pain Management Nursing 2000;1:13-21.

48. Chatelle C, Thibaut A, Bruno MA, Boly M, Bernard C, Hustinx R, Schnakers C, Laureys S. Nociception Coma Scale-Revised Scores correlate with metabolism in the anterior cingulate cortex. Neurorehabilitation and Neural Repair 2014;28:149-52.

49. The Multi-Society Task Force on PVS. Medical aspects of the persistent vegetative state (1). The New England Journal of Medicine 1994;330:1499-1508.

50. Schnakers C, Vanhaudenhuyse A, Giacino J, Ventura M, Boly M, Majerus S, Moonen G, Laureys S. Diagnostic accuracy of the vegetative and minimally conscious state: Clinical consensus versus standardized neurobehavioral assessment. BMC Neurology 2009; $9: 35$.

51. Demertzi A, Schnakers C, Ledoux D, Chatelle C, Bruno MA, Vanhaudenhuyse A, Boly M, Moonen G, Laureys S. Different beliefs about pain perception in the vegetative and minimally conscious states: A European survey of medical and paramedical professionals. Progress in Brain Research 2009;177: 329-338.

52. Pignolo L, Rogano S, Quintieri M, Leto E, Dolce G. Decreasing incidence of paroxysmal sympathetic hyperactivity syndrome in the vegetative state. Journal of Rehabilitation Medicine 2012;44: 502-504.

53. Bogner JA, Corrigan JD, Fugate L, Mysiw WJ, Clinchot D. Role of agitation in prediction of outcomes after traumatic brain injury. American Journal of Physical Medicine \& Rehabilitation 2001;80: 636-644.

54. Schnakers C, Zasler ND. Pain assessment and management in disorders of consciousness. Current Opinion in Neurology 2007;20: 620-626.

55. Borsook D. Neurological diseases and pain. Brain 2012;135: 320-344. 